\title{
Compartment Syndrome Following Total Knee Arthroplasty: Clinical Results of Late Fasciotomy
}

\author{
Cheol-Hee Park, MD, Seung-Hyuk Lee, MD, Dong-Geun Kang, MD, Kye-Youl Cho, MD, Sang-Hak Lee, MD, \\ and Kang-Il Kim, MD \\ Department of Orthopaedic Surgery, Center for Joint Diseases and Rheumatism, Kyung Hee University Hospital at Gangdong, Seoul, Korea
}

Compartment syndrome after total knee arthroplasty (TKA) is a rare complication. Because of its rarity, it may be overlooked and misdiagnosed as peroneal nerve palsy or deep vein thrombosis. This misdiagnosis could have a profound impact on the patient's outcome. We report a case of a 77-year-old female who developed unilateral compartment syndrome in the calf after staged bilateral TKA at an outside clinic. The patient presented with medical complications related to compartment syndrome: rhabdomyolysis and myoglobinuria, which caused acute renal failure. Thus, we performed late fasciotomy one week after symptom onset to debride necrotic tissue and salvage the compartment. In the discussion section, we will discuss risk factors for compartment syndrome after TKA, results of late fasciotomy and other indications for surgical treatment of compartment syndrome.

Keywords: Knee, Arthroplasty, Compartment syndromes, Complications

Compartment syndrome of the lower extremities is a dangerous condition, and in general, it is related to traumas such as contusions, fractures, and vascular damage ${ }^{1)}$. An important determinant for the successful treatment of compartment syndrome is an early diagnosis to ensure decompression of the pressure in the involved compartments. An early diagnosis and surgical decompression significantly affect the prognosis of the patient ${ }^{2-5)}$.

Recently, the incidence of complications, such as postoperative infection and implant loosening, has increased with the escalation in the number of total knee arthroplasties. In spite of this, compartment syndrome following total knee arthroplasty (TKA) is still an extremely rare complication ${ }^{4,6)}$. Because of its rarity, it

Received July 19, 2013; Revised December 14, 2013;

Accepted December 31, 2013

Correspondence to: Kang-Il Kim, MD

Department of Orthopaedic Surgery, Center for Joint Diseases and

Rheumatism, Kyung Hee University Hospital at Gangdong, 892

Dongnam-ro, Gangdong-gu, Seoul 134-727, Korea

Tel: +82-2-440-6151, Fax: +82-2-440-6296

E-mail: drkim@khu.ac.kr

This is an Open Access article distributed under the terms of the Creative Commons Attribution Non-Commercial License (http://creativecommons.org/licenses/by-nc/3.0/) which permits unrestricted non-commercial use, distribution, and reproduction in any medium, provided the original work is properly cited. may be overlooked and misdiagnosed as peroneal nerve palsy or deep vein thrombosis ${ }^{4}$. Unfortunately, this misdiagnosis could have a profound impact on the patient's outcome.

We report a case of a 77-year-old female who developed compartment syndrome after TKA and discuss risk factors for the condition, results of late fasciotomy, and other surgical indications for compartment syndrome.

\section{Case Report}

A 77-year-old female with acute renal failure was transferred to our emergency department from an outside clinic. The patient had diabetes mellitus and had been on oral hypoglycemic agents. Three weeks before transfer to our hospital, TKA of the left knee was performed under spinal anesthesia and 10 days before transfer, TKA of the right knee was performed under spinal anesthesia. During the left knee TKA, the patient had a huge blood loss and 4 pints of packed red blood cells (PRBCs) were transfused. After surgery, the patient's hemoglobin $(\mathrm{Hb})$ levels continued to fall, and thus 5 pints of PRBCs were transfused ( 2 pints, on the day of surgery; 1 pint, one day after surgery; and 2 pints, 1 week after surgery). At the time of the right knee TKA, there was also substantial bleeding, and 2 pints of PRBCs were transfused. The patient's postoperative $\mathrm{Hb}$ level continued to fall gradually and 2 
pints of PRBCs were additionally transfused on the postoperative day 2. Six days after the right knee TKA, the volume of urine was decreased to less than $500 \mathrm{~mL}$ per day. Laboratory findings showed acute renal failure [blood urea nitrogen (BUN), $35 \mathrm{mg} /$ $\mathrm{dL}$ (range, 6 to $22 \mathrm{mg} / \mathrm{dL}$ ); serum creatinine, $2.4 \mathrm{mg} / \mathrm{dL}$ (range, 0.6 to $0.9 \mathrm{mg} / \mathrm{dL}$ )], resulting in the transfer of the patient to our hospital.

After arrival in our emergency department, we conducted the same laboratory tests and confirmed that the patient was still in renal failure (BUN, $35 \mathrm{mg} / \mathrm{dL}$; serum creatinine, $2.2 \mathrm{mg} / \mathrm{dL}$ ) with a serum $\mathrm{Hb}$ level of $7.9 \mathrm{~g} / \mathrm{dL}$ (range, 12 to $16 \mathrm{~g} / \mathrm{dL}$ ). Rhabdomyolysis was also diagnosed: myoglobin, $372.8 \mathrm{IU} / \mathrm{L}$ (range, 11.1 to $57.5 \mathrm{IU} / \mathrm{L}$ ); creatine kinase, $1059 \mathrm{IU} / \mathrm{L}$ (range, 35 to $215 \mathrm{IU} / \mathrm{L}$ ); and lactate dehydrogenase, $1375 \mathrm{IU} / \mathrm{L}$ (range, 233 to $497 \mathrm{IU} / \mathrm{L}$ ).

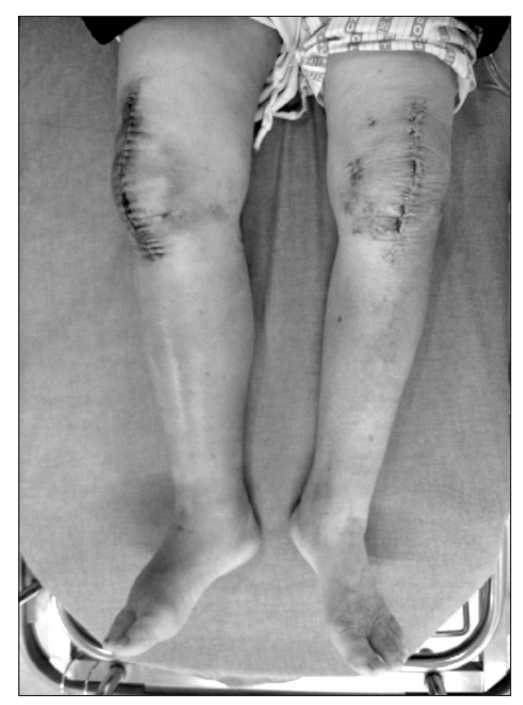

Fig. 1. The photograph shows more severe swelling in the right lower extremity than the left lower extremity.
We considered myoglobinuria due to rhabdomyolysis would be the cause of renal failure. In addition, coagulation tests revealed a prothrombin time of 29.1 seconds (range, 11.2 to $14.3 \mathrm{sec}$ ), an activated partial thromboplastin time of 47.6 seconds (range, 29 to $43 \mathrm{sec}$ ), an international normalized ratio of 2.96 (range, 0.84 to $1.16 \mathrm{sec}$ ), and a serum fibrinogen degradation products concentration of $82.5 \mathrm{~g} / \mathrm{mL}$ (range, 70 to $110 \mathrm{~g} / \mathrm{mL}$ ). Based on these findings, we inferred that consumptive coagulopathy was occurring.

Clinical examination of the right knee revealed that the operation wound had not yet healed. The wound was erythematous and slightly warm, but it was not severe and other clinical findings were not consistent with surgical site infection such as pus discharge. Compared to the left lower extremity, the right lower limb was more edematous with pitting edema (Fig. 1). Severe pain evoked by passive stretching of toes was complained. Below the right knee, the sensation was decreased to less than $50 \%$ of normal, and this paresthesia was not confined to a specific dermatome. She was unable to move her right ankle and foot voluntarily. The muscle power was grade zero for dorsiflexion and plantar flexion of the ankle and big toe. Color Doppler ultrasonography did not show any abnormalities of the posterior tibial artery or dorsalis pedis artery. The patient stated that when she recovered from spinal anesthesia on the operation day, severe pain was felt in the right foot with entire paresthesia below the right knee, and paralysis of the right ankle and foot developed. An interview with the primary care doctor of the referring hospital revealed that he had known about the paresthesia and paralysis, but had judged these findings had been caused by postoperative peroneal nerve palsy. Therefore, he only performed symptomatic treatment, including the use of analgesics, for severe pain.
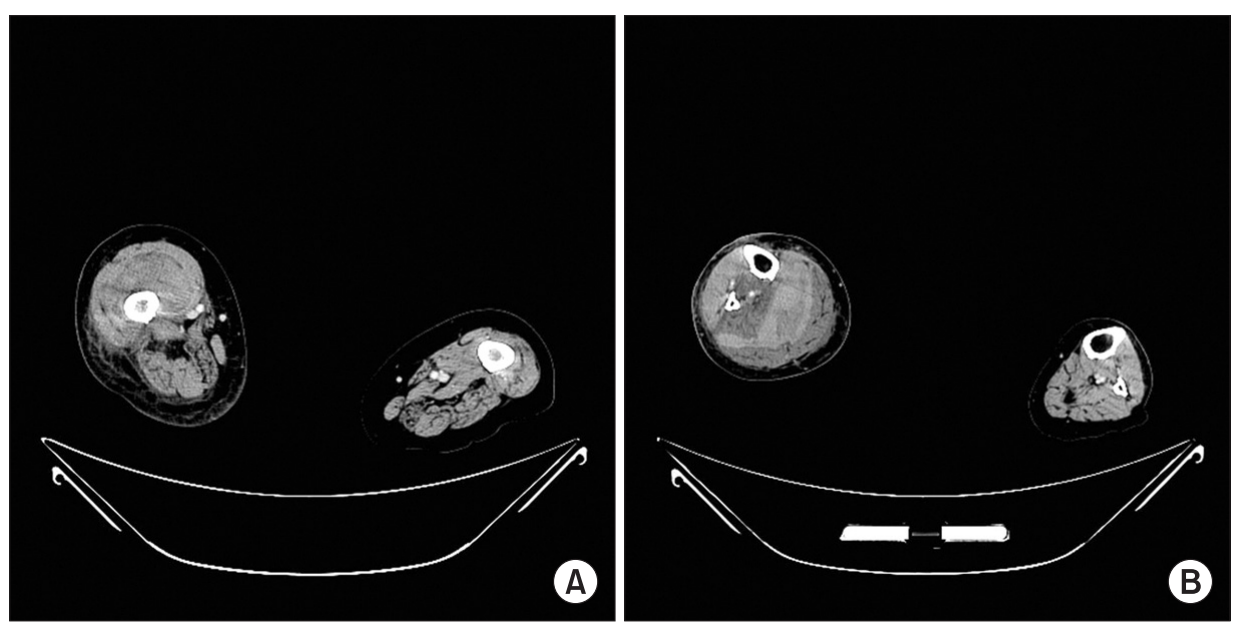

Fig. 2. Preoperative three-dimensional computer tomographic images show hematomas in the vastus intermedius and suprapatellar pouch (A) and in the posterior compartment of the leg (B). 
In consideration of the clinical findings, such as severe pain and edema of the right lower limb, paralysis and paresthesia not confined to specific neurological areas, and laboratory findings indicating rhabdomyolysis, we suspected compartment syndrome of the right lower extremity following TKA. Initially, we suspected a vascular injury and performed three-dimensional angiographic computerized tomography (CT). However, no vascular injuries or stenosis of the right lower limb were observed; massive hematomas were observed in the vastus intermedius above the right knee joint, suprapatellar pouch and anterior and posterior com-

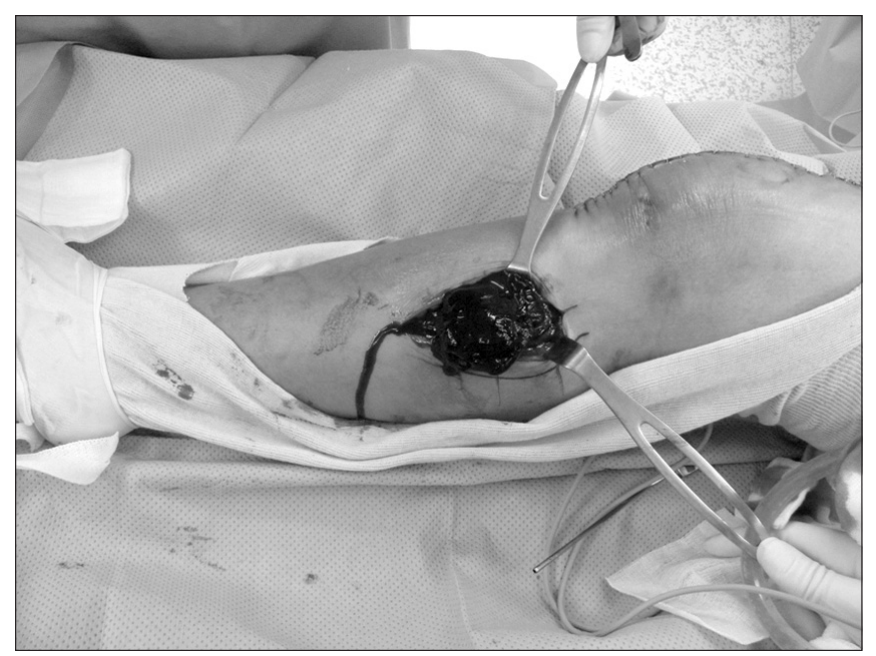

Fig. 3. The intraoperative photograph shows the posteromedial fasciotomy incision. The hematoma in the posterior compartment extruded immediately after fasciotomy.

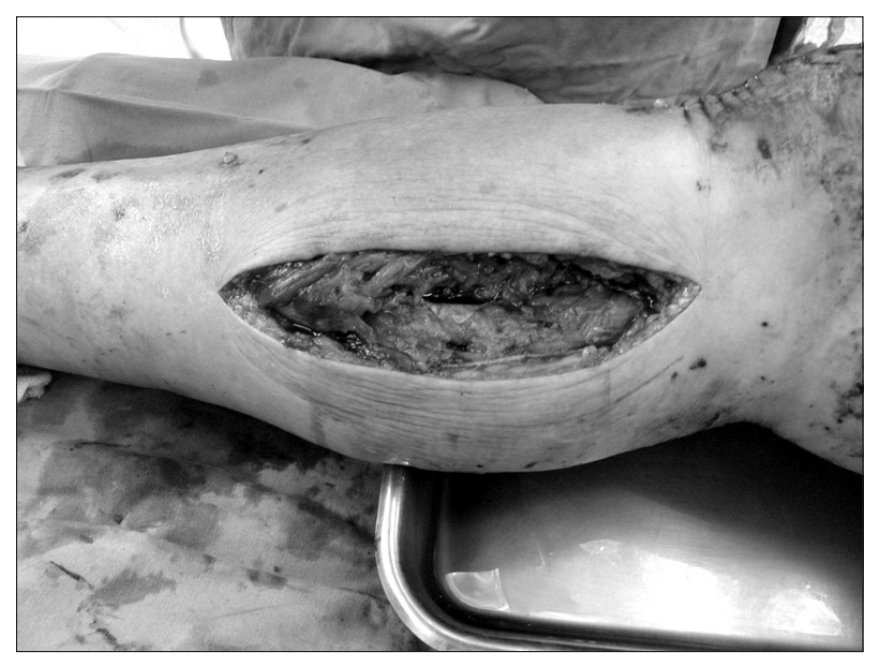

Fig. 4. The intraoperative photograph shows unviable tissues remaining after hematoma evacuation from the posterior compartment of the right leg. The muscle of the posterior compartment had unhealthy color, poor consistency, decreased contractility and inability to bleed. partments of the calf (Fig. 2). Based on these findings, we speculated that hematomas occurred after TKA because of persistent bleeding and, as a result, caused compartment syndrome of the right lower limb. To decompress the involved compartment and to remove necrotic tissue, we performed fasciotomy of the right lower extremity.

Fasciotomy was performed on the anterior, lateral, and posterior compartments of the calf through two anterolateral and posteromedial incisions. During surgery, we detected a massive hematoma in the posterior compartment. In particular, the he-

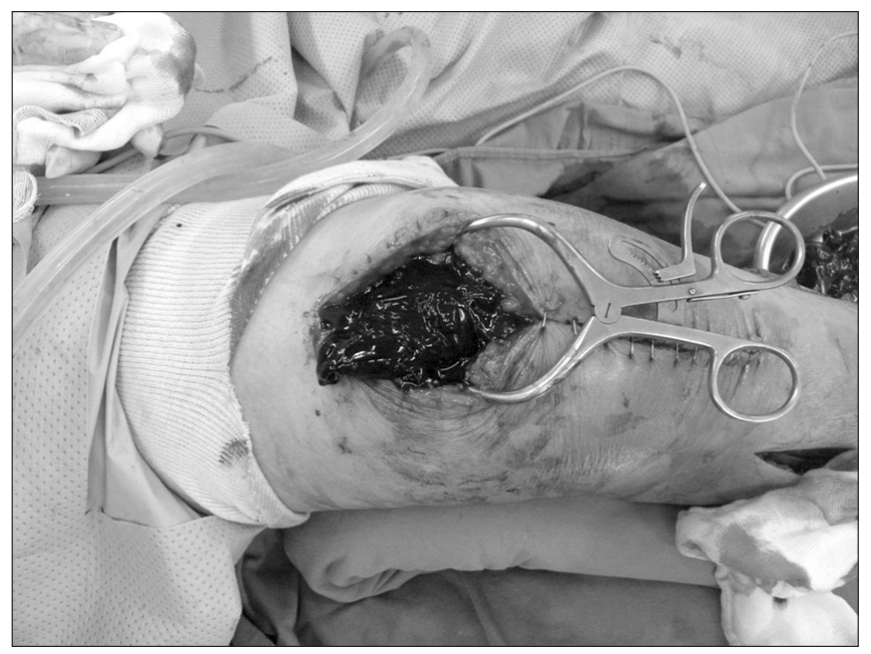

Fig. 5. Hematomas were observed in the vastus intermedius and suprapatellar pouch of the knee joint.

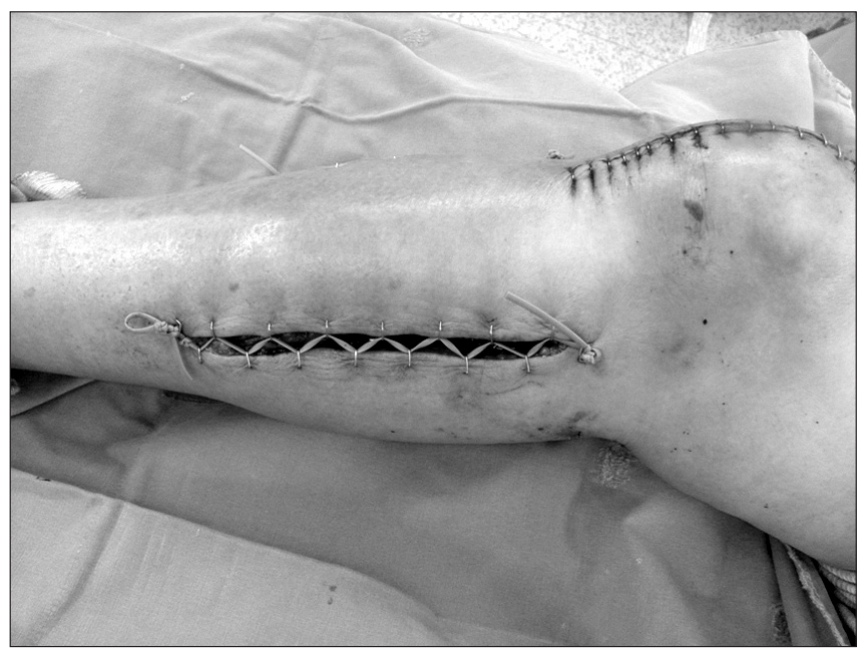

Fig. 6. After hematoma evacuation and tissue debridement, approximation suture using vessel loops and staples were applied with minimal tension. 
matoma of the posterior compartment extruded as soon as the fascial incision was made, indicating very high pressure in the posterior compartment (Fig. 3). Based on the color, consistency, contractility, and bleeding capacity of the posterior compartmental muscles, we diagnosed advanced necrosis of these muscles (Fig. 4). In contrast, the viability of the anterior compartmental muscles was relatively good compared to that of the posterior compartmental muscles. We removed all hematomas from the anterior and posterior compartments of the calf and debrided the necrotic muscles. Following fasciotomy, we opened the wound of the prior operation and found a massive hematoma in the vastus intermedius and knee joint (Fig. 5). We also observed extensive dissection of the periosteum and surrounding soft tissues of the distal femur at the operation site. However, there was no evidence of active bleeding in the prior surgical area. We removed hematomas from the vastus intermedius and suprapatellar pouch of the knee joint cavity and performed massive irrigation of the entire operation site to prevent infection. And the wound of the prior arthroplasty was securely sutured again. To maintain the decompression of compartmental pressure, the surgical wound of the calf was approximated with minimal tension for easy wound care (Fig. 6).

Pain in the ankle and foot disappeared immediately after surgery, and acute renal failure caused by myoglobinuria due to rhabdomyolysis improved within a week. However, distal sensory and motor ability of the right lower extremity did not recover immediately. We continued to administer antibiotics and sterilize the operation wound. At 3 weeks after surgery, the operation wound had improved so we debrided the remaining necrotic tissue and closed the fasciotomy wound. Currently, 2 years after fasciotomy, the patient can walk with mild difficulty in ankle motion, though a severe injury of the common peroneal nerve and tibial nerve was confirmed by electromyography and nerve conduction velocity.

\section{Discussion}

Compartment syndrome after TKA is extremely rare ${ }^{1,4,6)}$. It can occur in the calf and/or thigh compartments remote from TKA sites: the blood from bones and soft tissue usually collects in the joint cavity or is drained outside through a drainage tube $e^{1,4,6,7)}$. Although the incidence of compartment syndrome after TKA is very low, certain risk factors have been identified. These risk factors include bleeding due to extensive soft tissue dissection and hematoma formation, postoperative venous insufficiency through thrombus or direct major vessel injury, iatrogenic co- agulopathy resulting from an anticoagulant administration, unnecessarily high tourniquet pressure, a history of previous compartment syndrome, or previous surgery in the same $\mathrm{leg}^{1,4,5,8)}$.

In our case, TKAs were done under spinal anesthesia. After recovering from spinal anesthesia, even though there was severe pain with neurological symptoms in the lower extremity that underwent surgery, these symptoms were mistaken for peroneal nerve palsy. As a result, only symptomatic treatment was performed to control pain, and excessive analgesic administration made it difficult to diagnose compartment syndrome. After transfer to our hospital, three-dimensional enhanced CT angiography did not show findings consistent with direct damage of the blood vessels. However, the $\mathrm{Hb}$ level of the patient decreased continuously after arthroplasties, indicating that blood vessel damage could not be completely ruled out. In addition, after the left knee TKA, massive blood transfusion was performed due to persistent bleeding before the right knee TKA. This may have resulted in postoperative consumptive coagulopathy in combination with other risk factors such as surgery. Considering that an anti-thrombotic agent was not used after TKAs, delayed blood clotting may have caused continuous bleeding and then compartment syndrome. Furthermore, tourniquet pressure of $350 \mathrm{mmHg}$ in TKAs was unnecessarily high ${ }^{9)}$. In addition, at the time of fasciotomy, we observed extensive dissection of the periosteum and surrounding soft tissue of the distal femur and suspected an extensive soft tissue injury. These factors may also have contributed to the development of compartment syndrome ${ }^{8}$.

If compartment syndrome is diagnosed in the lower extremities, surgical treatment to reduce the pressure of the involved compartment, such as fasciotomy, should be performed urgently. Generally, it has been known that fasciotomy within 6 to 8 hours after the onset of symptoms could result in a good prognosis ${ }^{2-5)}$. Little or no return of function can be expected if diagnosis and treatment are delayed ${ }^{3}$. Because of the possibility of severe infection of the necrotic soft tissue through the operation wound, fasciotomy is generally contraindicated 8 to 35 hours after the onset of symptoms ${ }^{2,3,5)}$. If fasciotomy is not feasible, treatments that complement lost functions are recommended ${ }^{5}$ : use of an ankle brace, tendon transfer, and/or arthrodesis of the ankle joint may be considered as late treatment options. However, if there are medical complications related to compartment syndrome, for example, myoglobinuria due to rhabdomyolysis, late fasciotomy to debride necrotic tissue and salvage the compartment may be efficacious $^{5,10)}$. Williams et al. demonstrated that late fasciotomy performed 36 hours after symptom onset could result in limb salvage with good functional outcome in spite of increased infection 
rates $^{10)}$. Our patient had rhabdomyolysis and myoglobinuria due to the occurrence of compartment syndrome, which led to acute renal failure. Therefore, we performed late fasciotomy one week after symptom onset to relieve the medically compromised status. After the surgery, laboratory and clinical features of rhabdomyolysis and acute renal failure were markedly improved.

This report has several limitations. First, we did not measure the exact compartment pressure before fasciotomy. This was because we performed fasciotomy rapidly after transfer of the patient to our hospital: considering the patient's clinical symptoms, hematologic findings, and CT findings showing massive hematomas, compartment syndrome of the right lower leg, a condition that requires rapid surgical treatment, was strongly suspected. Surgical findings such as extrusion of the hematoma and muscle necrosis of the involved compartment supported our diagnosis. Second, we relied more on the medical records of the other hospital and interviews with the doctors that previously treated the patient than on direct observation to determine the patient's clinical symptoms after the right knee TKA.

In summary, compartment syndrome after TKA, albeit very rare, can significantly affect the prognosis of the patient. Therefore, it is important to be aware of the associated risk factors to prevent this potential complication, and, if it occurs, to ensure early diagnosis and prompt treatment to decompress the involved compartment and resolve the causative factor(s). If compartment syndrome is accompanied by medical problems such myoglobinuria due to rhabdomyolysis and acute renal failure, late fasciotomy with debridement of necrotic tissue should be considered as a treatment option, regardless of the time elapsed since the onset of symptoms.

\section{Conflict of Interest}

No potential conflict of interest relevant to this article was reported.

\section{References}

1. Boonstra RH, Haverkamp D, Campo MM, van der Vis HM. Acute compartment syndrome of the thigh following total knee arthroplasty. Knee. 2012;19:151-3.

2. Ebraheim NA, Abdelgawad AA, Ebraheim MA, Alla SR. Bedside fasciotomy under local anesthesia for acute compartment syndrome: a feasible and reliable procedure in selected cases. J Orthop Traumatol. 2012;13:153-7.

3. Finkelstein JA, Hunter GA, Hu RW. Lower limb compartment syndrome: course after delayed fasciotomy. J Trauma. 1996;40:342-4.

4. Haggis P, Yates P, Blakeway C, Fick D, Morgan DA, Holt M, Wood D. Compartment syndrome following total knee arthroplasty: a report of seven cases. J Bone Joint Surg Br. 2006;88:331-4.

5. Lasanianos NG, Kanakaris NK, Roberts CS, Giannoudis PV. Compartment syndrome following lower limb arthroplasty: a review. Open Orthop J. 2011;5:181-92.

6. Hailer NP, Adalberth G, Nilsson OS. Compartment syndrome of the calf following total knee arthroplasty: a case report of a highly unusual complication. Acta Orthop. 2007; 78:293-5.

7. Tang WM, Chiu KY. Silent compartment syndrome complicating total knee arthroplasty: continuous epidural anesthesia masked the pain. J Arthroplasty. 2000;15:241-3.

8. Nadeem RD, Clift BA, Martindale JP, Hadden WA, Ritchie IK. Acute compartment syndrome of the thigh after joint replacement with anticoagulation. J Bone Joint Surg Br. 1998; 80:866-8.

9. Hirvensalo E, Tuominen H, Lapinsuo M, Helio H. Compartment syndrome of the lower limb caused by a tourniquet: a report of two cases. J Orthop Trauma. 1992;6:469-72.

10. Williams AB, Luchette FA, Papaconstantinou HT, Lim E, Hurst JM, Johannigman JA, Davis K Jr. The effect of early versus late fasciotomy in the management of extremity trauma. Surgery. 1997;122:861-6. 